\title{
Our panel of experts highlight the most important research articles across the spectrum of topics relevant to the field of neurodegenerative disease management
}

Fiona Kumfor*,1,2,3 \& Olivier Piguet ${ }^{1,2,3}$

\section{Hughes LE, Rittman T, Regenthal R, Robbins TW, Rowe JB. Improving response inhibition systems in frontotemporal dementia with citalopram. Brain 138(7), 1961-1975 (2015).}

Frontotemporal dementia is a progressive dementia syndrome, for which no effective treatments or cures currently exist. In the behavioral-variant of frontotemporal dementia (bvFTD) disinhibition is one of the key clinical features. This study by Hughes et al. published in Brain, combined magnetoencephalography (MEG) and EEG in 12 bvFTD patients, to investigate the neurophysiological basis of behavioral disinhibition in these patients using a Go-NoGo paradigm. In a randomized double-blind placebo-controlled crossover study, the authors further sought to investigate whether a single dose of citalopram ameliorated abnormal behavioral disinhibition and its neural markers in these patients. The results showed that at the behavioral level, bvFTD showed worse inhibition (increased commission errors on NoGo trials) compared with controls. Moreover, EEG/MEG results revealed that bvFTD showed diminished neurophysiological responses, including in the right inferior frontal gyrus and right temporal pole, during successful inhibition. Notably, a trend for citalopram to partially restore these diminished neurophysiological responses was observed. No difference in behavioral performance, however, was seen between placebo and citalopram conditions. Although preliminary, these results suggest that citalopram may improve neural function in some bvFTD patients. Whether these neurophysiological findings can translate into improvement of behavioral and clinical symptoms remains to be seen, and warrants future investigation. Finally, the findings suggest that measurement of brain function using MEG/EEG can prove informative in these progressive neurodegenerative syndromes.

- Written by Fiona Kumfor

Butts AM, Machulda MM, Duffy JR, Strand EA, Whitwell JW, Josephs KA. Neuropsychological profiles differ among the three variants of primary progressive aphasia. J. Int. Neuropsychol. Soc. 21(6), 1-7 (2015).

Primary progressives aphasias (PPA) describe a range of brain disorders characterized by early and prominent language disturbance, against a background of relatively preserved other cognitive abilities. Three main syndromes are generally described based on the types of language deficits present: logopenic PPA, agrammatic PPA and semantic PPA but distinction among these syndromes remains difficult in the clinic, given their overlapping language features. In this study, the authors examined the cognitive performance of 91 patients diagnosed with PPA (51 logopenic, 27 agrammatic and 13 semantic) and matched on demographic variables and disease severity. They hypothesized that the groups would also differ on non-language cognitive tests (memory, processing speed, executive 
function, visuospatial ability). Analyses showed that the logopenic PPA group tended to perform the worst overall on cognitive tests, even after taking into account aphasia severity. In addition, each group showed cognitive deficits that were consistent with their respective pattern of underlying brain atrophy, with memory and visuospatial impairment suggestive of temporoparietal involvement in logopenic PPA, executive dysfunction mediated by left prefrontal region in agrammatic PPA and naming and verbal memory denoting left anterior temporal involvement in semantic PPA. This study adds to the accumulating evidence demonstrating that patients with PPA experience cognitive deficits beyond the language sphere, including social cognition as reported elsewhere. Together, this information will help improve diagnostic accuracy, particularly between the two nonfluent language presentations, logopenic and agrammatic PPA, where language features are often difficult to distinguish.

- Written by Olivier Piguet

\section{Financial \& competing interests disclosure}

The authors have no relevant affiliations or financial involvement with any organization or entity with a financial interest in or financial conflict with the subject matter or materials discussed in the manuscript. This includes employment, consultancies, honoraria, stock ownership or options, expert testimony, grants or patents received or pending, or royalties.

No writing assistance was utilized in the production of this manuscript. 